University of Nebraska - Lincoln

DigitalCommons@University of Nebraska - Lincoln

4-6-2017

\title{
Unexpected Nongenetic Individual Heterogeneity and Trait Covariance in Daphnia and Its Consequences for Ecological and Evolutionary Dynamics
}

Clayton E. Cressler

Stefan Bengtson

William A. Nelson

Follow this and additional works at: https://digitalcommons.unl.edu/bioscifacpub

Part of the Biology Commons

This Article is brought to you for free and open access by the Papers in the Biological Sciences at DigitalCommons@University of Nebraska - Lincoln. It has been accepted for inclusion in Faculty Publications in the Biological Sciences by an authorized administrator of DigitalCommons@University of Nebraska - Lincoln. 


\title{
Unexpected Nongenetic Individual Heterogeneity and Trait Covariance in Daphnia and Its Consequences for Ecological and Evolutionary Dynamics
}

\author{
Clayton E. Cressler, ${ }^{1}$ Stefan Bengtson, ${ }^{2}$ and William A. Nelson ${ }^{2, \star}$ \\ 1. School of Biological Sciences, University of Nebraska, Lincoln, Nebraska 68588; 2. Department of Biology, Queen's University,
} Kingston, Ontario, Canada

Submitted May 18, 2016; Accepted December 22, 2016; Electronically published April 6, 2017

Online enhancements: appendixes, $\mathrm{R}$ code.

\begin{abstract}
AвStract: Individual differences in genetics, age, or environment can cause tremendous differences in individual life-history traits. This individual heterogeneity generates demographic heterogeneity at the population level, which is predicted to have a strong impact on both ecological and evolutionary dynamics. However, we know surprisingly little about the sources of individual heterogeneity for particular taxa or how different sources scale up to impact ecological and evolutionary dynamics. Here we experimentally study the individual heterogeneity that emerges from both genetic and nongenetic sources in a species of freshwater zooplankton across a large gradient of food quality. Despite the tight control of environment, we still find that the variation from nongenetic sources is greater than that from genetic sources over a wide range of food quality and that this variation has strong positive covariance between growth and reproduction. We evaluate the general consequences of genetic and nongenetic covariance for ecological and evolutionary dynamics theoretically and find that increasing nongenetic variation slows evolution independent of the correlation in heritable life-history traits but that the impact on ecological dynamics depends on both nongenetic and genetic covariance. Our results demonstrate that variation in the relative magnitude of nongenetic versus genetic sources of variation impacts the predicted ecological and evolutionary dynamics.
\end{abstract}

Keywords: demographic heterogeneity, individual stochasticity, lifehistory covariation, individual-based model, stoichiometric food quality, Daphnia pulicaria.

\section{Introduction}

Rarely do all individuals in a population have the same life history. At any snapshot in time, individuals can have different genetics, be exposed to different resources and envi-

\footnotetext{
* Corresponding author; e-mail: nelsonw@queensu.ca. ORCIDs: Cressler, http://orcid.org/0000-0002-6281-2798.
}

Am. Nat. 2017. Vol. 190, pp. E13-E27. (C) 2017 by The University of Chicago. 0003-0147/2017/19001-56994\$15.00. All rights reserved. DOI: $10.1086 / 691779$ ronments, be at different ontogenetic stages, or have different maternal histories. These processes generate variation in vital rates among individuals that is collectively referred to as individual heterogeneity (Conner and White 1999). When individual heterogeneity in vital rates occurs in a population, it generates structure within a population that is referred to as demographic heterogeneity (Kendall and Fox 2002; Benton et al. 2006; Melbourne and Hastings 2008). A well-studied example of demographic heterogeneity is ontogenetic stage structure, which emerges from the individual heterogeneity caused by differences in vital rates between juvenile and adult individuals (de Roos and Persson 2013). For individual heterogeneity to give rise to demographic heterogeneity, the vital rate differences among individuals must persist for some period of time (Kendall and Fox 2003; Bolnick et al. 2011; Stover et al. 2012). The specific structure of demographic heterogeneity in a population depends on how the underlying vital rate variation was created. Some sources of demographic heterogeneity create temporary groups with similar vital rates, such as individuals with the same maternal history (e.g., Beckerman et al. 2002) or cohorts of individuals born on the same day (Fox et al. 2006). Other sources create groups that persist indefinitely, such as those created by genetic differences (Kendall et al. 2011) or ontogenetic stage structure (de Roos and Persson 2013).

Observations of the life-history traits of individuals can be used to characterize individual heterogeneity in a population (e.g., Cam et al. 2002), but a more challenging question is whether this individual heterogeneity impacts ecological or evolutionary dynamics (Vindenes et al. 2008; Vindenes and Langangen 2015). One approach has been to correlate individual life-history traits with population dynamics in systems where both longitudinal and population data are available. For example, the repeated crashes in Soay sheep densities are explained by the interaction of 
weather with the demographic age and sex structure of the population (Coulson et al. 2001; Pelletier et al. 2007), and the alternating cycles of dwarfism and gigantism in Eurasian perch emerge from the interaction between sizedependent cannibalism and the size structure of the population (Persson et al. 2000). Another approach is to combine population models based on life-history traits with population-scale experiments. Most of this work has been done in taxa where the vital rates are strongly stage structured (e.g., juvenile vs. adult) and has revealed that this individual heterogeneity can have a significant impact on dynamics, such as generating large-amplitude cycles (Briggs et al. 2000; Persson et al. 2007; Nelson et al. 2013) and creating alternative states in a system (Claessen et al. 2000; McCauley et al. 2008).

These case studies demonstrate the ecological importance of vital rate heterogeneity that generates age or stage structure. However, individuals often have lifetime differences in vital rates that are unrelated to age or stage. Recent theory suggests that such lifetime differences in vital rates can also have a strong impact on biological dynamics. For example, vital rate heterogeneity among individuals can increase population growth rates and equilibrium densities (Kendall et al. 2011; Stover et al. 2012) and reduce extinction risk in stochastic models (Conner and White 1999; Kendall et al. 2002; Singh et al. 2004; Melbourne and Hastings 2008; Acker et al. 2014).

An important distinction is whether individual variation in vital rates is due to genetic differences among individuals or whether the source of heterogeneity is nongenetic. This distinction is, of course, important for evolutionary dynamics, but it is also important for ecological dynamics (Vindenes and Langangen 2015). Consider a nongenetic example where the source of variation is caused by differences among cohorts. If a cohort of individuals has a larger body size on average than the previous cohort, perhaps owing to a better environment for their mothers, these individuals can have higher survivorship, growth, and reproduction over their entire lives if their body size enables them to gain access to more resources (van Noordwijk and de Jong 1986). This cohort then becomes a group in the population with different vital rates than other groups, but the group only lasts as long as the cohort is alive. In contrast, if genetic variation is responsible for demographic heterogeneity, then the population can be structured into genotypes (e.g., homozygotes and heterozygotes), with offspring having a probability of being added to one group versus others based on heredity (e.g., Kendall et al. 2011). Since individuals are being continuously added to a genotype group, the group will likely last longer than groups based on differences among cohorts. Thus, whether individual heterogeneity has a genetic basis is likely to have different impacts on ecological and evolutionary dynamics.
Here we use life-history experiments to study the individual heterogeneity from both genetic and nongenetic sources in a species of freshwater zooplankton (order Cladocera). Even under constant environmental conditions, cladocerans have a tremendous amount of vital rate variation (Lynch 1988; Tessier and Consolatti 1989) generated from two sources: developmental noise (Yampolsky and Scheiner 1994; Olijnyk and Nelson 2013) and genotypic variation (Baird et al. 1990; Epp 1996; Latta et al. 2015). Owing to the asexual life cycle of species in this order, this system provides a rare opportunity to characterize the variation from genetic versus nongenetic sources of demographic heterogeneity. Theory suggests that the ecological or evolutionary response depends on the covariance structure among traits (Lindström and Kokko 2002; Benton et al. 2006; Kendall et al. 2011). Our experiments allow us not only to characterize the variance in life-history traits but also to characterize the covariance among traits from both nongenetic and genetic sources of individual heterogeneity. As previous work has shown that trait covariance can change across environments (Sgrò and Hoffmann 2004), we experimentally created a gradient of food quality and observed the response of individual heterogeneity in life-history traits due to both genetic and nongenetic sources. We choose stoichiometric food quality (measured as the ratio of carbon to phosphorus) because it has a strong impact on zooplankton life history (Sterner and Hessen 1994) and is a relevant environmental gradient in natural systems (Elser et al. 2009).

The experiments reveal a surprising amount of nongenetic variation among life-history traits that is unexpectedly different in both magnitude and covariance structure from genetic variation. Using the experimental results as a basis, we develop a general mathematical model to evaluate the consequences of individual heterogeneity from genetic versus nongenetic sources for ecological and evolutionary dynamics across a wide range of covariance structures. We find that nongenetic individual heterogeneity can increase population growth rates while simultaneously reducing the rate of adaptive evolution. This combination of experimental and theoretical results thus provides direct empirical evidence for differences in genetic and nongenetic sources of individual heterogeneity in a wellstudied model organism and the potential consequences of that heterogeneity for ecological and evolutionary dynamics.

\section{Methods \\ Source Animals}

We used the freshwater zooplankton Daphnia pulicaria because it has a cyclic parthenogenetic life cycle that 
allows for the experimental separation of genetic and nongenetic effects and because it displays an abundance of nongenetic life-history variation (Olijnyk and Nelson 2013). Our source population is from Round Lake, Ontario, Canada ( 15 ha; $\left.44^{\circ} 32^{\prime} \mathrm{N}, 76^{\circ} 24^{\prime} \mathrm{W}\right)$, where we collected samples of live zooplankton on May 5, 2011. Isofemale lines were reared in the laboratory using COMBO medium (Kilham et al. 1998) at $20^{\circ} \mathrm{C}$ and fed the green alga Scenedesmus acutus. Of the original 80 isofemale lines isolated from the lake, 55 were cultivable in the laboratory. Genotypes were identified using cellulose acetate electrophoresis (Hebert and Beaton 1993), which revealed nine multilocus genotypes using the phosphoglucose isomerase, phosphoglucomutase, lactate dehydrogenase, and malate dehydrogenase loci. A single isofemale line was selected from each of the nine multilocus genotypes to use in the experiments.

\section{Experiments}

We performed a series of individual-scale life-history experiments across a wide range of food quality. The experiments used a fully crossed design with nine genotypes in each of five food-quality environments with a minimum of 30 replicate individuals in each treatment (table A1; tables A1A4, C1, C2 are available online). All individuals were fed the green alga $S$. acutus. Individual Daphnia were kept in the dark in $35-\mathrm{mL}$ vials with $20 \mathrm{~mL}$ of algal food and COMBO medium and provided a food ration of $0.05 \mathrm{mg}$ of carbon every 2 days. This method was developed to control individual resource ingestion, which allows us to study trait variation without the confounding effect of differential resource ingestion (Olijnyk and Nelson 2013). The food ration was created by diluting stock algae with nitrogen- and phosphorus-free COMBO medium. Every second day, individuals were measured for length and reproductive output and transferred to a clean vial with a new food ration. Measurements continued for the full life span of each individual. Length measurements were obtained using a dissecting microscope (Leica MZ6) under 32× magnification, calibrated each measurement day with a stage micrometer. Individuals were measured from the base of the tail spine to the top of the head. The number of eggs and/or neonates observed released into the water at each transfer were recorded as the measure of reproductive output.

To evaluate how variation and covariation among lifehistory traits changed with environmental stress, we conducted the experiments across a range of stoichiometric food-quality levels. The food-quality gradient was created by manipulating the carbon-to-phosphorus $(\mathrm{C}: \mathrm{P})$ ratio of the algae. We first cultured $S$. acutus under conditions that resulted in very poor quality and then supplemented the algae with different amounts of monobasic potassium phosphate $\left(\mathrm{K}_{2} \mathrm{HPO}_{4}\right.$; Plath and Boersma 2001). Phospho- rus addition was done $90 \mathrm{~min}$ prior to feeding, which takes advantage of the ability for P-limited S. acutus to take up most of the dissolved $\mathrm{P}$ in under $30 \mathrm{~min}$ (Lehman and Sandgren 1982; Plath and Boersma 2001). Stock cultures of $S$. acutus were raised in batch culture using autoclaved $\left(121^{\circ} \mathrm{C}, 30 \mathrm{~min}\right) \mathrm{COMBO}$ medium at $23^{\circ} \mathrm{C}$ with $\sim 500 \mu \mathrm{moL}$ of photons $\mathrm{m}^{-2} \mathrm{~s}^{-1}$ and a daily light regime of 18L:6D. The COMBO medium was as given in Kilham et al. (1998) but with $0.75 \mu \mathrm{M} \mathrm{K}_{2} \mathrm{HPO}_{4}$ and $75 \mu \mathrm{M} \mathrm{NaNO}$. Cultures were harvested 10 days after inoculation and were found to have an average $\mathrm{C}: \mathrm{P}$ of 1,133:1 ( $\pm 4 \mathrm{SE}$ ). Each batch was inoculated with a volume of the previous culture calculated to provide a constant inoculation density of cells, which helped maintain consistency in algal population growth. Cell density in the cultures was estimated by counting a 1:10-diluted Lugol's solution-stained algal culture sample in a 1-mL Sedgwick-Rafter counting chamber. Algal cells in 18 haphazardly chosen squares of the slide were counted using a compound microscope (Olympus CX21) at 100x magnification. Densities from a minimum of two independently diluted counts were used to estimate cell density in the culture flasks. To convert to carbon, we used a value of 30.82 pg of $\mathrm{C}$ per cell based on $\mathrm{CHN}$ combustion analysis.

We conducted the experiments at five food-quality levels: C:P of $200: 1,400: 1,600: 1,800: 1$, and 1,000:1. This range spans much of the natural variation of particulate $\mathrm{C}: \mathrm{P}$ found in small lakes (Guildford and Hecky 2000) and provides environments that are both above and below the threshold phosphorus ratio for Daphnia at the food concentration provided (Urabe and Watanabe 1992). To check that life-history responses were due to changes in food quality rather than digestibility caused by morphological changes in algal cells (e.g., van Donk et al. 1997), we fed the poorest-quality algae to a set of trial individuals and examined their lower gut contents. We found no undigested cells, indicating that indigestibility was not a factor at the food-quality levels used in our experiment. To check the amount of food ration consumed after 2 days, we preserved the vial contents from 92 individuals across all food-quality levels and counted the density of algae cells remaining. Average consumption was $95 \%$, with most (91\% of the individuals) consuming $>90 \%$ of the available food algae.

\section{Analysis of Life-History Traits}

We used three scalar measures to capture the life-history patterns of growth, reproduction, and longevity. For individual growth, we first converted the every-other-day measurements of length $(L)$ in millimeters to length-based carbon weight $(w)$ in micrograms using the relationship $w=\xi L^{q}$, where $\xi=2.63$ and $q=2.4$ (Paloheimo et al. 
1982). The conversion to length-based weight allows ease of comparison between growth and reproduction but has no impact on the statistical results (app. A; apps. A-F are available online). For individuals surviving longer than 9 days, individual weight trajectories were fit with a generalized additive model (GAM) with a monotonic increasing constraint (Pya and Wood 2015). This approach allows for the statistical characterization of growth performance without imposing a predescribed shape to the growth pattern. We used the mean mass gain between age 0 and 10 days from the fit GAM as the scalar measure for growth $(\kappa)$. Early-life growth performance was a good predictor of lifetime growth performance (fig. A1; figs. A1-A4, B1, C1, C2, D1-D5, E1, F1 are available online), and this approach avoids any potential spurious correlation with age of death. A similar approach (fitting a GAM to cumulative reproduction) was used to calculate the individual reproductive rate during the first 10 days $(\omega)$ after reaching size at maturity (Ebert 1994). Longevity was measured as the age of death $(\delta)$. Fits were done using the scam package (Pya and Wood 2015) in the R software environment ( $\mathrm{R}$ Core Team 2014; app. A).

Owing to the asexual nature of $D$. pulicaria, the abovedescribed scalar measures of growth, reproduction, and longevity can be used to distinguish the amount of individual heterogeneity from genetic sources versus nongenetic sources. For growth and reproduction, we can also estimate the amount of individual stochasticity. The method for parsing the observed life history into different sources of variation follows from the definition of each. Individual heterogeneity is the variation in vital rates among individuals that persist throughout an individual's lifetime (Kendall and Fox 2002). Individual stochasticity, in contrast, is the day-to-day variation in realized life-history values of an individual caused by chance events for a given set of lifetime vital rates (Caswell 2009). By fitting the GAM described above to data on age-specific size (or cumulative reproduction) of each individual, we characterize the expected growth (or reproduction) trajectory resulting from a fixed set of expected life-history traits for an individual. Since the deterministic part of the model averages over the day-to-day variation in realized life history caused by individual stochasticity, it allows us to statistically separate individual heterogeneity from individual stochasticity. Variation in the deterministic growth (or reproduction) among individuals within a genotype provides an estimate of nongenetic sources of individual heterogeneity, and the variation among genotypes provides an estimate of genetic sources of individual heterogeneity. The variation is then calculated for each source using the scalar measures of growth, reproduction, and longevity as described above.

The strength of individual stochasticity can be estimated for growth and reproduction using the residual variation around the fit deterministic model of each individual. The stochastic model is

$$
Y_{t}=Y_{t-1}+g_{t}+s_{t}
$$

where $Y_{t}$ is the predicted individual size (or cumulative reproduction) at age $t, g_{t}$ is the amount of deterministic growth (or reproduction) over the age interval, and $s_{t}$ is individual stochasticity.

The age interval is set at 2 days to match the data. Deterministic growth (or reproduction) $g_{t}$ is calculated from the deterministic GAM $\left(y_{t}\right)$ fit to age-specific weight (or cumulative reproduction) as

$$
g_{t}=y_{t}-y_{t-1}
$$

Stochastic growth $s_{t}$ is represented by an autoregressive integrated moving average (ARIMA) model fit to the residual variation around the growth (or reproduction) trajectory of each individual. ARIMA models are time-series models that are ideal for growth (or reproduction) trajectories because they allow estimation and simulation of the serial dependence in the residuals. The stochastic growth (or reproduction) model is given as

$$
s_{t}=\operatorname{ARIMA}(p, d, q),
$$

where $p$ is the number of autoregressive terms, $d$ is the order of differencing, and $q$ is the number of moving average terms. Since growth (or reproduction) trajectories differ among individuals, the most appropriate ARIMA model in terms of $p, d$, and $q$ will also differ (details in app. B). Individual stochasticity was estimated for each individual from 500 parametric bootstrap simulations of the stochastic growth model (fig. B1). We assume that all residual variation is the result of individual stochasticity rather than observation error, which means that our estimate of individual stochasticity is an upper estimate. Using an overestimate of individual stochasticity means that the conclusions we draw about the comparatively small magnitude of individual stochasticity are conservative. All analyses were done in the $\mathrm{R}$ software environment ( $\mathrm{R}$ Core Team 2014) using the arima() and arima.sim() functions.

To study the covariance among life-history traits emerging from genetic versus nongenetic sources of individual heterogeneity, we estimate the genetic and nongenetic covariance using a multivariate generalized linear mixed model with genotype as a random effect (Hadfield 2010). Each food level was analyzed separately to estimate how these matrices change across the environmental gradient. The replicate individuals within each genotype allow us to isolate the nongenetic covariance from the genetic covariance. A positive genetic covariance-for example, be- 
tween growth and reproduction-suggests that genotypes with consistently faster growth rates also have consistently higher reproductive rates. A positive nongenetic covariance, on the other hand, suggests that individuals with fast growth rates also have high reproductive rates independent of genotype. Fits were done using the MCMCglmm package (Hadfield 2010) in the R environment (R Core Team 2014), which uses Markov chain Monte Carlo (MCMC) methods to fit multivariate generalized linear mixed models. Code specifying the MCMC run details and the prior specification can be found in appendix C. ${ }^{1}$ We verified that our choice of prior had no effect on the statistical conclusions.

\section{Ecological and Evolutionary Consequences of Individual Heterogeneity}

We used an individual-based model to study the consequences of genetic and nongenetic covariance on ecological and evolutionary dynamics (app. D). An individual-based model is advantageous because it allows us to simultaneously account for both sources of trait variation among individuals: individual heterogeneity from genetic variation and individual heterogeneity from nongenetic variation. We characterize each individual (i) by its genotype, current stage (juvenile or adult), and expected daily rates of birth $\left(\beta_{i}\right)$, mortality $\left(\delta_{i}\right)$, and maturation $\left(\mu_{i}\right)$. The parameters representing the expected rates of birth, mortality, and maturation are fixed for an individual when they are born, which means that variation among individuals in their expected rates is the source of demographic heterogeneity in the model. To study the ecological consequences of individual heterogeneity, we simply track the population dynamics of the system. To study the evolutionary consequences, we quantify the fitness variance among the genotypes in the population. The fitness of each genotype is its population growth rate $\left(R_{i, t}=N_{i, t+1} / N_{i, t}\right)$, where $N_{i, t}$ is the number of individuals of genotype $i$ at time $t$. The fitness variance determines the magnitude of evolutionary change due to natural selection in classic population genetic theory (Fisher 1930), so we can use the relative magnitude of fitness variance to predict how genetic and nongenetic heterogeneity impact the magnitude or rate of evolutionary change due to natural selection.

For both genetic and nongenetic forms of individual heterogeneity, we consider all possible combinations of negative $\left(G_{1}\right)$, uncorrelated $\left(G_{2}\right)$, and positive $\left(G_{3}\right)$ matrices that describe the covariance among the life-history traits of birth, longevity, and maturation:

1. Code that appears in The American Naturalist is provided as a convenience to the readers. It has not necessarily been tested as part of the peer review.

$$
\begin{aligned}
& G_{1}=\left[\begin{array}{ccc}
1 & -0.5 & -0.5 \\
-0.5 & 1 & -0.5 \\
-0.5 & -0.5 & 1
\end{array}\right], \\
& G_{2}=\left[\begin{array}{lll}
1 & 0 & 0 \\
0 & 1 & 0 \\
0 & 0 & 1
\end{array}\right], \\
& G_{3}=\left[\begin{array}{ccc}
1 & 0.5 & 0.5 \\
0.5 & 1 & 0.5 \\
0.5 & 0.5 & 1
\end{array}\right] .
\end{aligned}
$$

Each simulation was initialized with 100 genotypes drawn from a trivariate normal distribution of life-history traits with mean zero and covariance given by one of the matrices described above. We converted these standardized trait values to a biologically relevant scale using the trait means and standard deviations observed at the highest food level (fig. 2). This allows us to use the standard multivariate normal distribution to specify covariances instead of needing to account for differences in trait means and variances. While each run of the simulation generates a new set of random genotypes, the genotypes are fixed for the duration of a simulation. Once the genotypes are created, the next step is to create the individuals. Individuals within each genotype are created by sampling a multivariate normal distribution with mean equal to the genotype's life-history traits and covariance given by one of the matrices described above, multiplied by a scalar that allows us to control the amount of nongenetic variation. This allowed us to investigate how increasing the amount of nongenetic variation affects ecological and evolutionary dynamics. Simulations were started with 20 adults of each genotype and the dynamics updated daily as follows for 100 days, which represents roughly 14 generations (based on the average maturation time of 7.1 days). At every daily time step, each individual in the population could die, survive as a juvenile, survive and transition from juvenile to adult, or survive as an adult and reproduce. Survival was decided first on the basis of the outcome of a Bernoulli trial, with probability based on the individual-specific daily mortality rate as $1 / \delta_{i}$. If the individual survived and was a juvenile, it could mature to the adult stage based again on the outcome of a Bernoulli trial, with probability based on the individualspecific development rate as $\mu_{i}$. If the individual survived and was an adult, it could reproduce based on a random draw from a Poisson distribution, with expectation given by the reproduction parameter $F_{i}$. The reproduction parameter includes the daily birth rate that characterizes the individual $\left(\beta_{i}\right)$ that is then adjusted for density dependence as

$$
F_{i}=\beta_{i} \exp (-\alpha N)
$$


(e.g., Neubert and Caswell 2000), where $N$ is the total population size summed across all genotypes and $\alpha$ controls the strength of density dependence. Since our primary interest is in studying the role played by nongenetic versus genetic individual heterogeneity, the model does not include individual stochasticity. We set the strength of density dependence $\left(\alpha=1 \times 10^{-4}\right)$ to give equilibrium densities that were sufficiently large to ensure that demographic stochasticity was negligible. Results are shown as the average of 50 simulations for all combinations of genetic and nongenetic covariance matrices and for six levels of nongenetic trait variance.

\section{Results}

We found genotypic variation in all life-history traits across all food treatments (fig. 1). While the genotypes studied in our experiments were only a subset of those found in our source lake, the genetic variation that we observed is comparable to the genetic variation observed among genotypes hatched from resting eggs, which represents the potential genetic variation in a lake (table A3;
Ebert 1991). Surprisingly, we found considerable variation in growth and reproduction among individuals within a genotype (fig. 1). In fact, the variance among individuals within a single genotype is substantially larger than the variance among the genotypes studied (fig. 2). Even as the food environment degrades to a point where reproduction has almost shut off, the within-genotype variance is greater for all traits (fig. C1). Since individuals are clonal, these results reveal that the magnitude of individual heterogeneity attributable to nongenetic sources is much greater than genetic sources in this species. The estimated strength of individual stochasticity is comparable to observed genetic variation, which is much smaller than the variation from individual heterogeneity (fig. 2).

Without distinguishing the source of the variation, simple correlations among life-history traits suggest a positive correlation between growth and reproduction (fig. A3). By distinguishing genetic from nongenetic sources of individual heterogeneity, we find that none of the genetic trait correlations are significantly different from zero (fig. 3), which means that genotypes that invest a lot in any particular life-history trait do not invest less (or more) in other
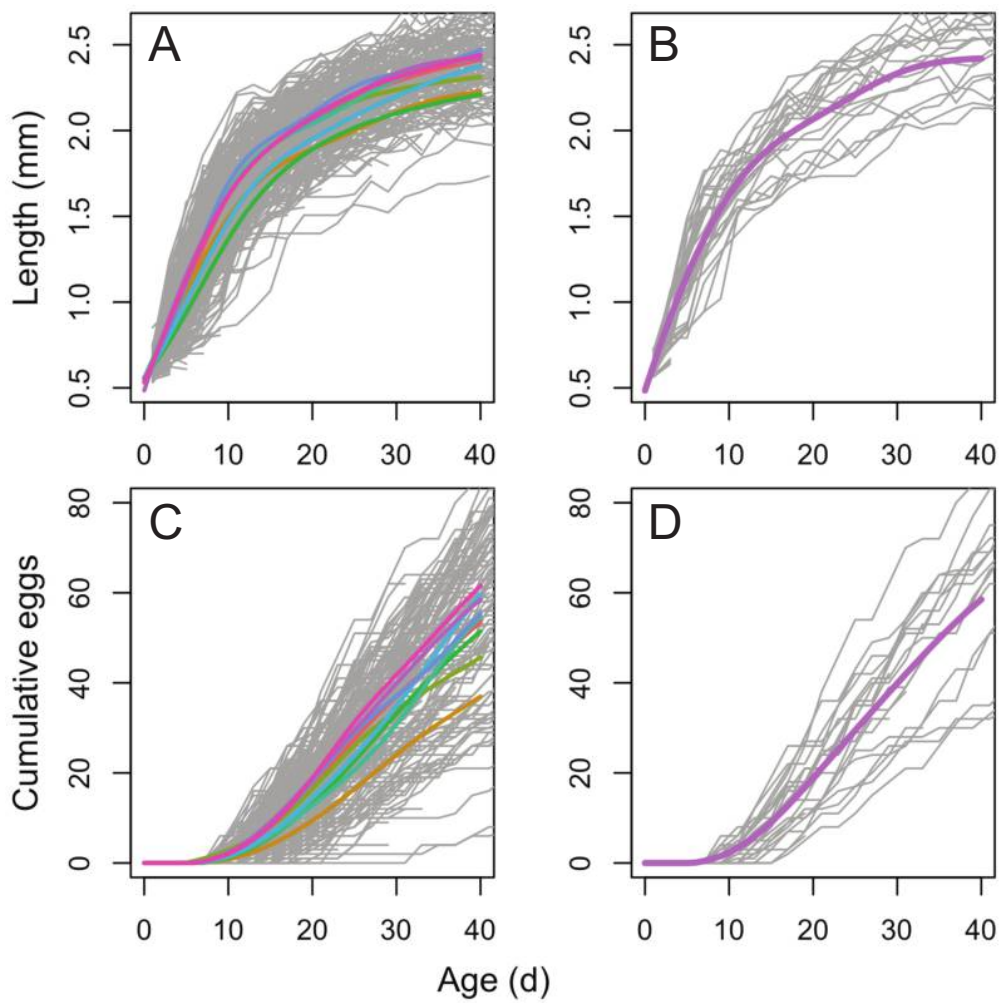

Figure 1: Observed growth $(A)$ and reproduction $(C)$ trajectories by age for each individual (gray lines) at the highest food-quality level. The end of the line indicates the age of death. Mean trajectories for each genotype (colored lines) are shown as the fit of a generalized additive model to all individuals of the genotype. To illustrate the typical variation among individuals within a genotype, $B$ and $D$ show individual trajectories for one of the genotypes from $A$ and $C$ (purple lines), respectively. 

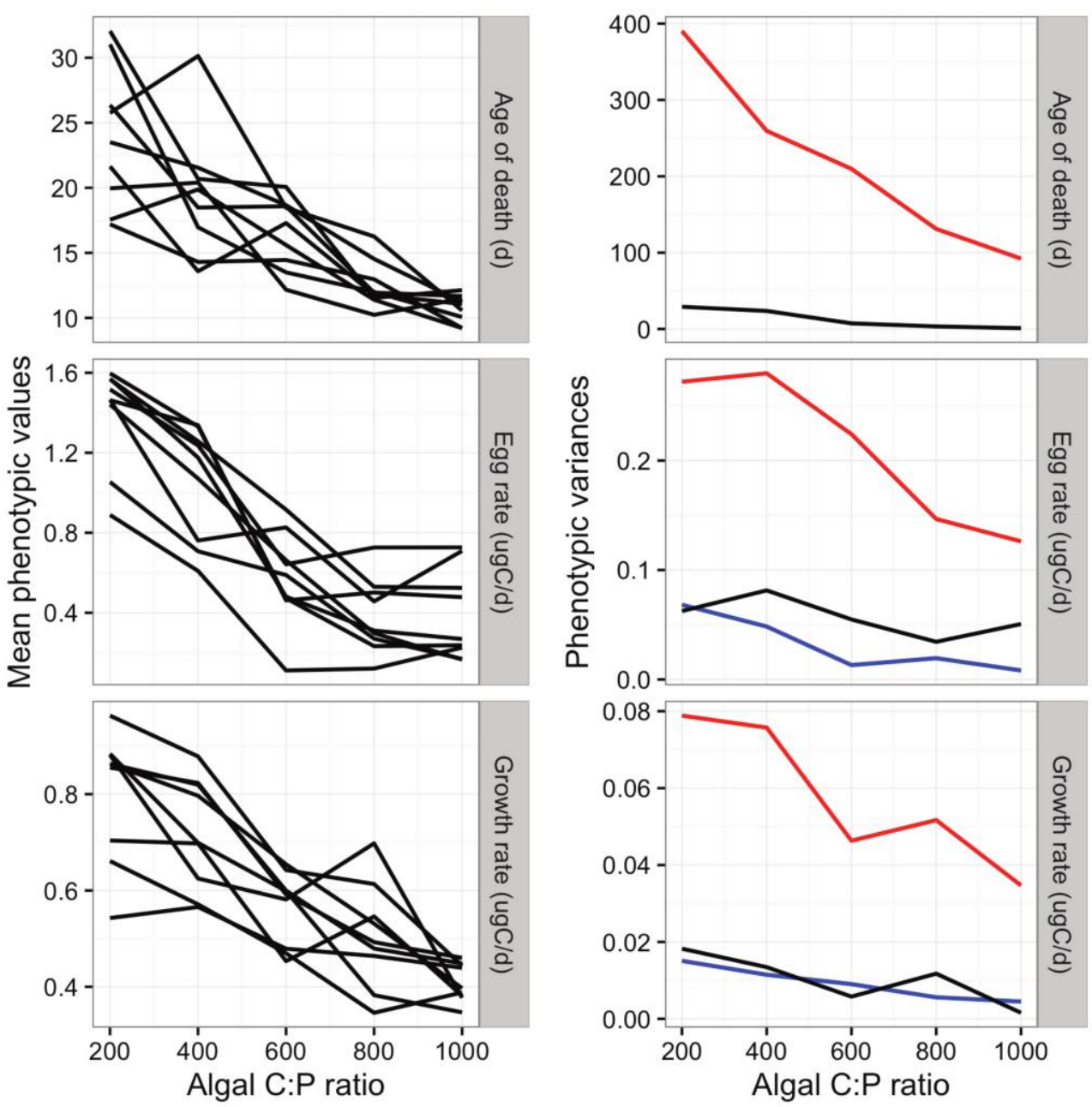

Figure 2: Mean trait values for each genotype across a gradient in food quality (left column). The lines in each panel denote a different genotype. The right-hand panels show direct estimates of the trait variance among individuals within each genotype (red lines) and trait variation among genotypes (black lines), which reveals that the variation within a genotype is greater than the variation among genotypes for all traits and food levels. The estimated magnitude of individual stochasticity (blue lines) is similar to the trait variance among genotypes and is much less than the trait variation among individuals within a genotype.

traits (i.e., there is no evidence of trade-offs). In contrast, there is strong evidence for positive correlations among life-history traits for individuals within each genotype (fig. 3). This is especially true for the correlation between growth and reproduction, which is statistically positive for all but the lowest food quality. This indicates that when individuals have high growth rates, they also have high reproduction even though they are genetically identical and raised under carefully controlled food environments. To evaluate whether these results were sensitive to having higher levels of replication within a genotype than among genotypes, we conducted two evaluations of the method (app. F). In the first, we simulated data assuming only genetic covariance among the traits and estimated the falsepositive rate for detecting nongenetic covariance. In the second, we repeated the analysis with balanced replication by using subsets of the within-genotype data. The evaluation revealed a false-positive rate of $5 \%$, which is the same as the type I error rate used in the analysis, and that using subsets of the data with balanced replication resulted in the same conclusions as those shown in figure 3 . Taken together, the evaluation indicates that the statistical method has a high probability of distinguishing the covariance structure from genetic versus nongenetic sources of individual heterogeneity and showed that the method is not sensitive to having unbalanced replication. None of the nongenetic correlations were negative, indicating an absence of trade-offs among the traits.

To investigate the potential ecological and evolutionary implications of trait variation from the different sources, 


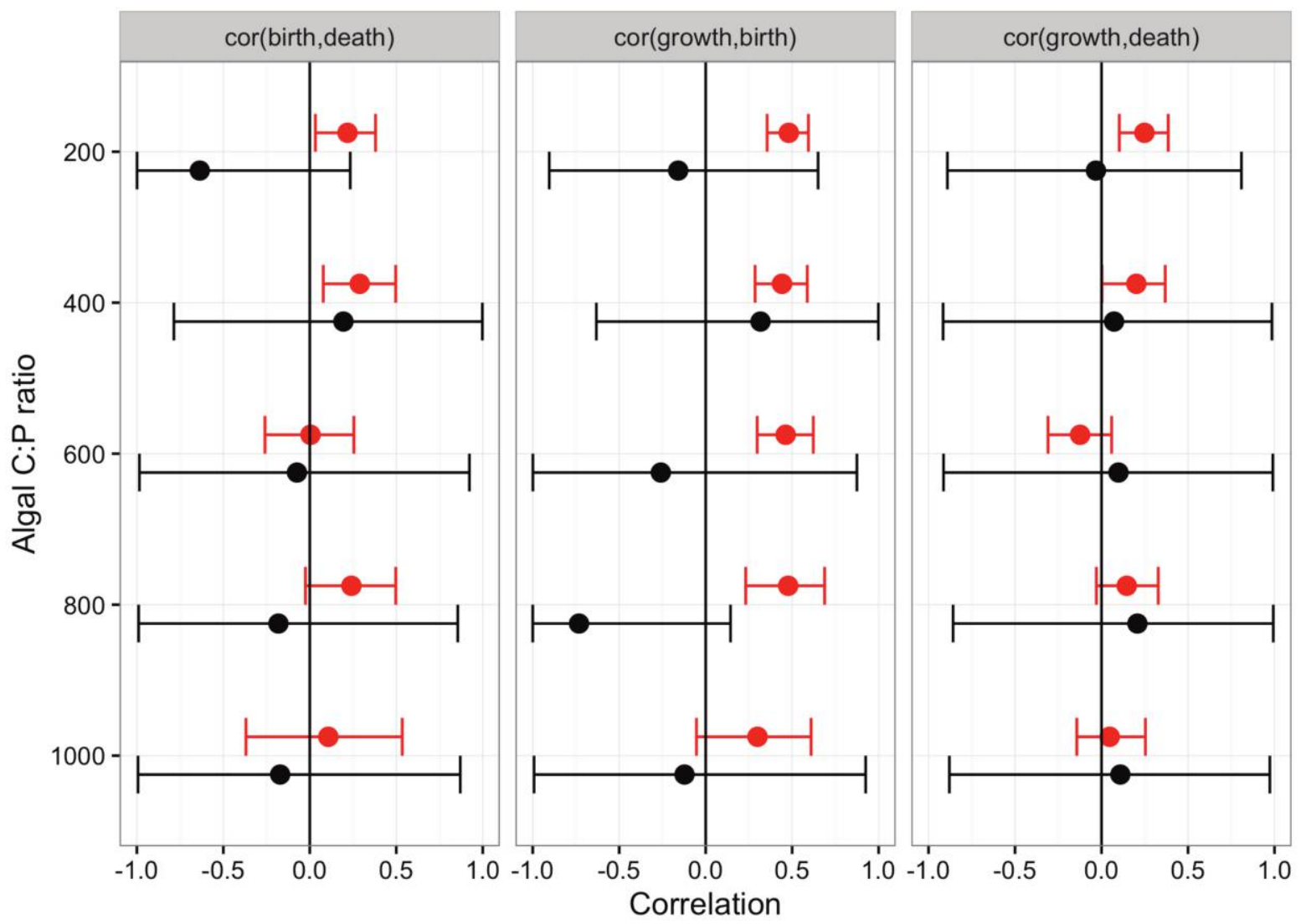

Figure 3: Correlations among life-history traits for variation from genetic sources (black) and nongenetic sources (red) across a gradient of decreasing food quality. Each vertical panel shows a different trait pair, and the error bars represent $95 \%$ confidence intervals.

we developed a population model that incorporates both genetic and nongenetic sources of individual heterogeneity. The direction of the ecological response to increasing nongenetic variation depended on the nongenetic covariance matrix but not on the genetic covariance matrix (fig. 4). If nongenetic covariation is negative, increasing nongenetic trait variation decreases population growth and the final density. If nongenetic covariation is zero or positive, increasing nongenetic trait variation increases population growth and the final density.

Nongenetic sources of variation also had a strong impact on fitness differences among genotypes. Increased levels of nongenetic variation-regardless of the covariation structure-always resulted in reduced fitness variance among the genotypes and thus a slower rate of evolution from natural selection (fig. 5). This is illustrated nicely by comparing the fitness for a set of genotypes in the absence versus presence of nongenetic variation (fig. D1). Genotypes with low fitness in the absence of nongenetic variation perform comparatively better when nongenetic variation is added than do genotypes with high fitness; this causes a reduction in the overall fitness variance among genotypes and correspondingly results in slower rates of evolution. The effect is amplified when the nongenetic covariance structure is negative because genotypes with high fitness in the absence of nongenetic variation have reduced fitness when nongenetic variation is added. The degree to which fitness variance is reduced by nongenetic variation depends on the combination of covariance structures: with positive genetic covariance, the greatest reduction in the fitness variation among genotypes occurs with positive nongenetic covariance; with negative genetic covariation, the greatest reduction in the fitness variation among genotypes occurs with negative nongenetic covariance (fig. 5).

Reducing food quality had large negative effects on all traits (fig. 2; table A2), with all genotypes showing a significant decline in at least two traits with decreasing food quality (table A4), and generally led to a loss of the positive covariance structure (fig. 3). To investigate the contribution of each source of individual heterogeneity for the predicted ecological and evolutionary dynamics in our experiments, we conducted a sensitivity analysis of the model using our empirically estimated genetic and nongenetic covariance matrices across the range of food quality. For the 


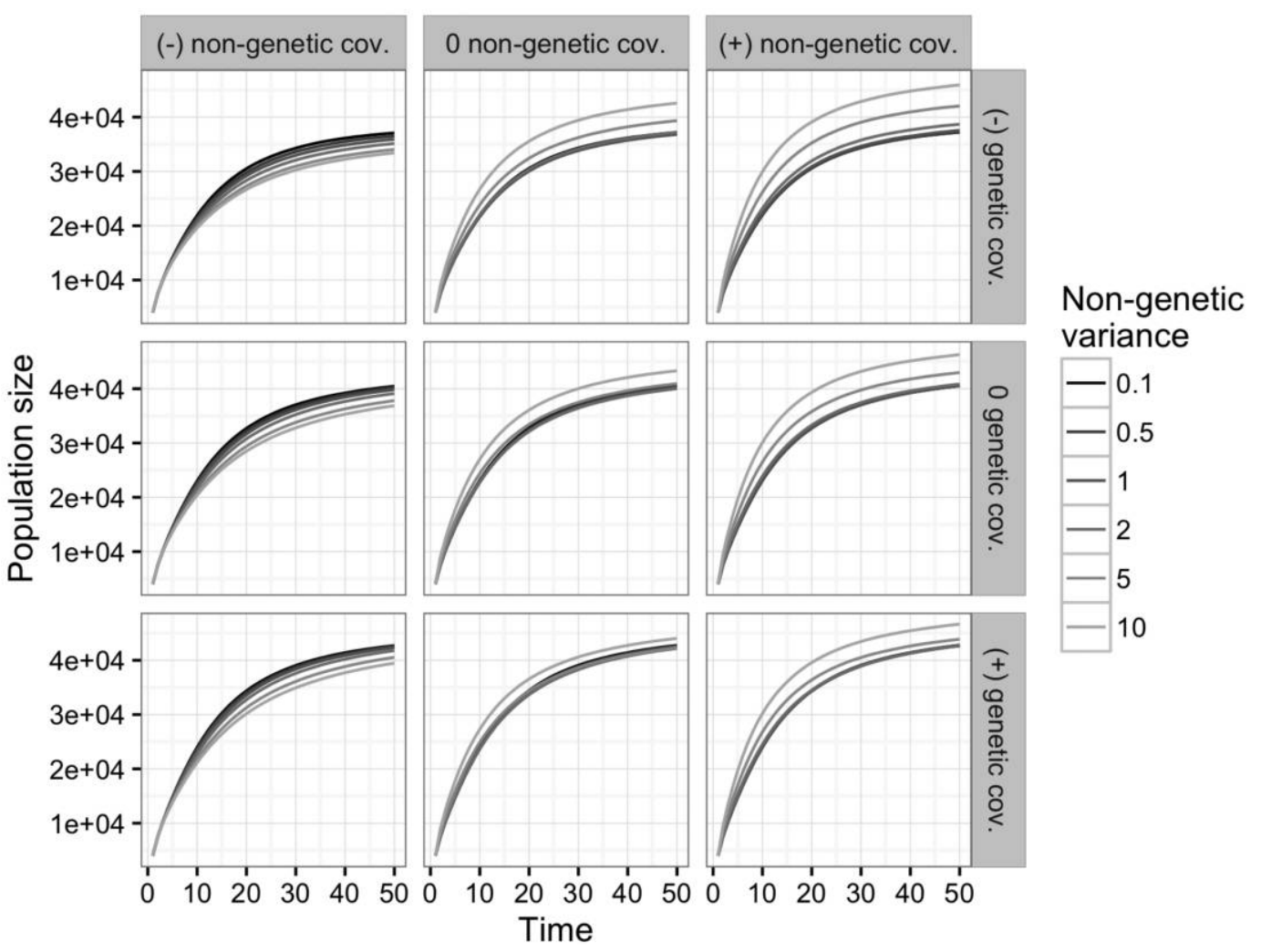

Figure 4: Predicted ecological impact of nongenetic variance. Increasing the amount of nongenetic variation has a strong influence on population growth rate and population size across all covariance structures (compare gray lines in each panel). If the nongenetic covariance is zero or positive (middle and right columns), increasing nongenetic variation increases population growth rates and the asymptotic population size. If the nongenetic covariance is negative (left column), increasing nongenetic variation decreases population growth rates and the asymptotic population size. The sign of any genetic covariance has a quantitative impact on population growth rate and asymptotic size, but it does not cause a qualitative change.

sensitivity analysis, we increased either genetic or nongenetic variation by $10 \%$ (i.e., we multiplied every term of the covariance matrix by 1.1) and calculated the percent change in population growth rate or fitness variance due to this increase. Predicted population growth rates decreased with decreasing food quality as expected, and increasing variation for both sources tended to increase the population growth rate (fig. $6 A, 6 B$ ). Increasing nongenetic variation had a larger effect on population growth than did increasing genetic variation. Fitness variance increased as food quality declined, suggesting that selection was stronger in poorer environments (fig. $6 \mathrm{C}$ ). Increasing trait variation had opposing effects on selection depending on the source of the variation. Increasing genetic variation always increased fitness variance, as expected, whereas increasing nongenetic variation always reduced fitness variance (fig. 6D). Genetic variation is predicted to have a larger relative impact on selection than nongenetic variation among the genotypes in our study. However, on average the nongenetic variation still contributed around half the relative impact on selection than genetic variation, meaning that both sources are predicted to play an important role in determining selection.

\section{Discussion}

Vital rate variation in populations is well documented (Bolnick et al. 2011), and theoretical work has shown that this variation can scale up to impact ecological (Grimm and Uchmański 2002; González-Suárez et al. 2011) and evolutionary (Metcalf and Pavard 2007; Vindenes and Langangen 2015) dynamics, even when that variation does not have any clear structure, such as that caused by ontogeny, gender, or size (Kendall et al. 2011; Stover et al. 2012). For example, individual heterogeneity in the flour beetle model system generates variation in demography that is detectable in time-series data (Melbourne and Hastings 2008), and individual heterogeneity in red deer explains a substantial amount of the observed population dynam- 


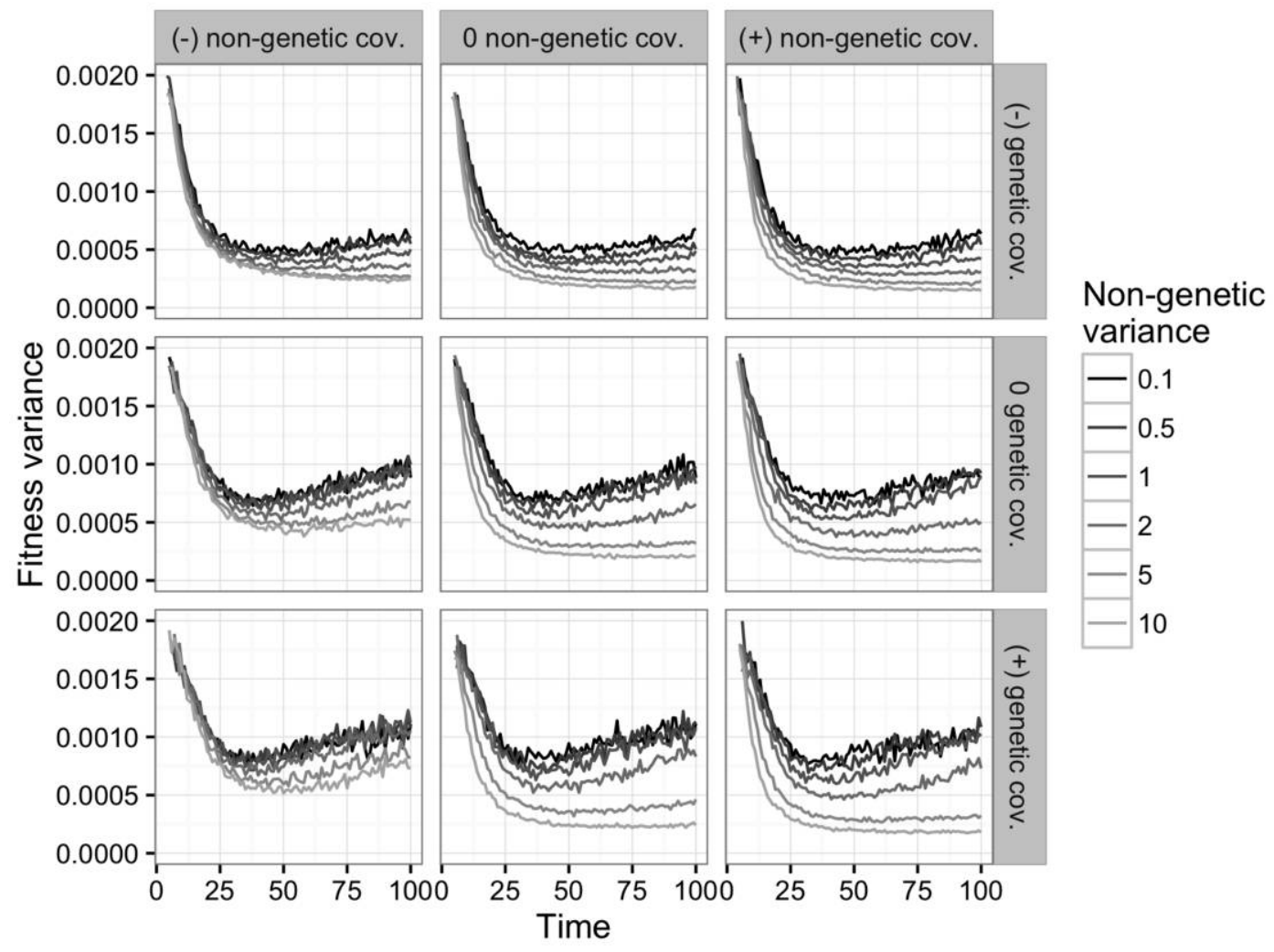

Figure 5: Predicted evolutionary impact of nongenetic variance. Increasing the amount of nongenetic trait variation always reduces the variance in fitness among the genotypes, thereby slowing the rate of evolution due to natural selection, no matter the genetic or nongenetic trait covariance structure.

ics (Pelletier et al. 2012). As a literary term, individual heterogeneity is useful because it distinguishes persistent vital rate differences among individuals from those differences due, for example, to demographic stochasticity. However, since different sources of individual heterogeneity generate different patterns of demographic structure in a population, there is need to identify and characterize vital rate variation from different sources and to predict how each scales up to impact ecological and evolutionary dynamics (Benton et al. 2006). In this study, we use a parthenogenetic organism to study the individual heterogeneity emerging from genetic versus nongenetic sources and use an individualbased model to predict the effect of each on ecological and evolutionary dynamics.

While previous work in Daphnia has found variation in life-history traits among individuals within a genotype (Lynch 1988; Olijnyk and Nelson 2013), our study is the first with sufficient replication to enable the estimation of the covariance among vital rates at both the withingenotype and the among-genotype level. We found substantial within-genotype variation in life-history traits across all food treatments. More interesting, however, is that this nongenetic variation is substantially larger than the trait variation among genotypes isolated from the same lake (fig. 2), indicating that nongenetic sources of individual heterogeneity contribute more variation than genetic sources. At first blush, that result might not seem surprising because there is an expectation that the heritability of life-history traits should be low (e.g., Price and Schluter 1991). This has been attributed to low additive genetic variation for life-history traits because of their close connection to fitness (Mousseau and Roff 1987) or to high levels of environmental variation that impact lifehistory traits, causing an elevation of nongenetic variation (Price and Schluter 1991). However, the magnitude of genetic variation observed in our experiments (table A3) is similar to median levels of genetic variation for traits that are not directly related to life history (Hansen et al. 2011). Moreover, since our experiments were conducted under tightly controlled environmental conditions, the amount of trait variation that can be attributed to the environment is greatly reduced relative to natural systems. Despite typical levels of genetic variation and tight environmental control, we find that nongenetic sources of variation, such as 

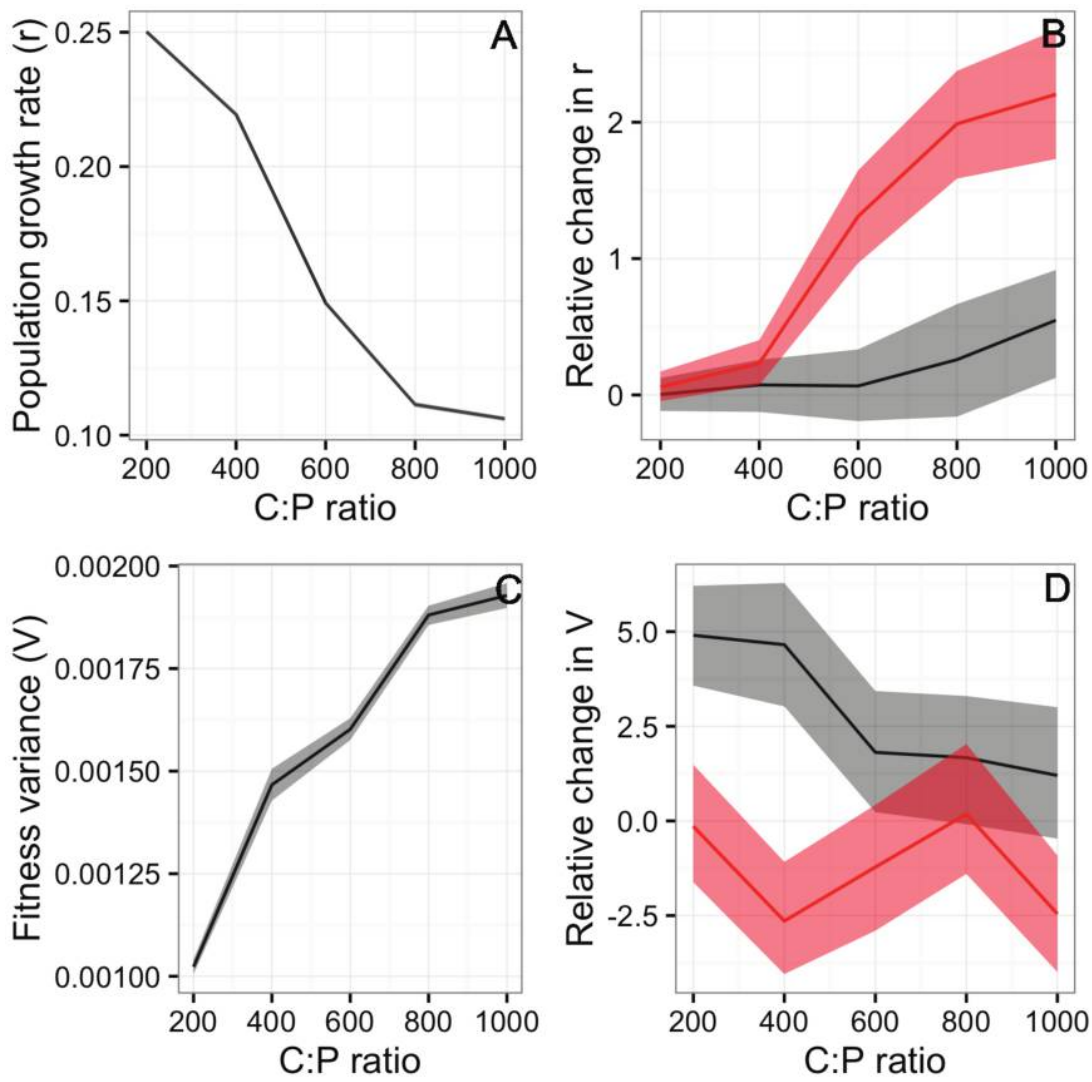

Figure 6: Predicted contribution from nongenetic and genetic heterogeneity in Daphnia based on empirically estimated trait means and covariance. A, Predicted population growth rate across food quality (high quality is $200 \mathrm{C}: \mathrm{P}$, low quality is $1,000 \mathrm{C}: \mathrm{P}$ ). $B, \mathrm{Relative}$ change (percent) in growth rate when nongenetic (red) versus genetic (black) variation is increased in each food environment, based on a sensitivity analysis. $C$, Predicted fitness variance across food quality. $D$, Relative change (percent) in fitness variance when nongenetic (red) versus genetic (black) variation is increased in each food environment, based on a sensitivity analysis.

developmental noise, are a more significant source of lifehistory variation than genetic sources.

The genotypes used in our experiments were isolated from a single location on a single day. While this is the relevant context for considering the impact of demographic heterogeneity on population dynamics, we need to consider whether these results are restricted to our population. Fortunately, there is a long history of research looking at the life-history differences among genotypes in Daphnia. We found six studies where data could be extracted for both the within-genotype and the among-genotype variance in life-history traits for a range of Daphnia species (Vanni 1987; Lynch 1988; De Meester 1995; Epp 1996; Barata and Baird 1998; Pietrzak 2011). While these studies cannot be used to analyze the covariance structure among life-history traits, as we have done here, they can be used to compare the variation from genetic versus nongenetic sources. These studies reveal that nongenetic variation was larger than genetic variation in $75 \%$ of the traits studied. Combined with the results of our experiments, there is strong evidence that the variation from nongenetic sources of individual heterogeneity is typically larger than intraspecific genetic variation in Daphnia.

While relatively rare, a handful of laboratory studies in other animal systems also provide data to evaluate the relative magnitude of genetic versus nongenetic sources of individual heterogeneity. In gastropods, nongenetic variation accounted for more of the variation in body growth than did genetic variation (Forbes et al. 1995). In Artemia, among-individual variation was found to be larger than among-genotype variation for traits related to reproduction and life span (Browne et al. 2002). In rotifers, some species have within-genotype variation in proportion of mixis offspring that is similar in magnitude to the variation among genotypes (Gilbert and Schroder 2007). These studies are not a comprehensive evaluation, but they demonstrate that nongenetic variation can be a strong and underappreciated source of individual heterogeneity. 
By carefully following the growth and reproduction trajectories of individual animals, our study is able to compare the strength of individual heterogeneity with the strength of individual stochasticity. Individual stochasticity is the random variation in vital rates among individuals and is distinct from the persistent differences that come from individual heterogeneity (Caswell 2009). In our experiments, the variation from individual stochasticity was much smaller than the variation from individual heterogeneity (fig. 2). This is perhaps not surprising for individuals raised in a carefully controlled laboratory environment; however, it suggests that individual heterogeneity can be as strong - and potentially stronger - than individual stochasticity. The relative strength of the different sources of variation has direct implications for the development of population models because each source is incorporated differently. Trait variation from individual stochasticity does not create any additional heterogeneity structure in a population. As such, it can be incorporated into population models using stochastic models that have the same trait structure as a model without any variation (e.g., Caswell 2009). In contrast, the variation from individual heterogeneity creates additional structure in the population because trait differences persist for a period of time. Incorporating demographic heterogeneity requires an explicit representation of the trait heterogeneity (Vindenes et al. 2008; Kendall et al. 2011; van Daalen and Caswell 2015). Pragmatically, it is easier to incorporate the variation from individual stochasticity because the only additional information required is the trait distribution, whereas incorporating individual heterogeneity requires knowledge of not only the trait distribution but also where the trait differences originate (e.g., reproduction vs. growth) and how long these differences persist. Despite this challenge, our results suggest that individual heterogeneity may be a more relevant source of variation for organisms than individual stochasticity and should receive more attention in the development of population models than it currently does.

An unexpected result from our experiments is that the relationships among traits are highly constrained for nongenetic trait variation (fig. 3). Specifically, we find strong evidence of positive life-history correlations among the traits describing reproduction, growth, and survivorship in an experimentally controlled environment that prevents differential ingestion of resources. The implication is that some individuals within a genotype have better performance than others across all life-history traits. The positive trait covariance found here is similar to previous work in this species (for discussion of mechanisms, see Olijnyk and Nelson 2013). The lack of life-history constraints among genotypes goes against the conclusions of previous work in a closely related species (Spitze et al. 1991; dis- cussed in Reznick et al. 2000), which is likely due to differences between our study designs. Spitze et al. (1991) used only a few individuals per genotype to characterize genetic variation. We show in appendix $E$ that with only a few individuals per genotype, positive nongenetic trait correlations can be mistakenly attributed to genetic sources. On the other hand, with the number of genotypes and individuals per genotype that we had in our experiment, it is very unlikely to mistakenly attribute positive trait correlations that are caused by genetics to nongenetic sources (app. F). As a result, we can conclude that the positive genetic correlations observed by Spitze et al. (1991) are plausibly the misidentification of positive nongenetic correlations.

The evolutionary response to increased nongenetic variation in our model always resulted in reduced fitness differences among genotypes and, hence, a reduced rate of evolution (fig. 5). The reduction comes from two mechanisms. The first is a form of the frailty effect (e.g., Kendall et al. 2011). Within any population, there will be individuals that are born particularly frail, with life-history traits that greatly reduce their development, reproduction, or survivorship. Since these individuals do not survive long, the distributions of the viable individuals will have a higher expected value than the distribution at birth. This effect increases the mean fitness of individuals in the presence of trait variation compared with the absence of trait variation. Fitness variation is reduced among a set of genotypes because the effect is greatest for genotypes thaton average-have low performance (figs. D1, D2). The second mechanism is from the nonlinear dependence of fitness on life-history traits. While increasing any single life-history trait will increase fitness, it always does so with diminishing returns (Boyce 1977), which means that fitness is guaranteed to be a concave function of life-history traits. As a result, mean fitness among individuals in the presence of variation is always lower than that in the absence of variation owing to the mathematical effect of Jensen's inequality. In particular, fitness differences among a set of genotypes will be reduced when the fitness of highperforming genotypes decreases more than it does for low-performing genotypes (figs. D1, D3). These two mechanisms combine to slow the rate of evolution by reducing the fitness of high-performing genotypes and increasing the fitness of low-performing genotypes in the presence of nongenetic trait variation (fig. D1).

Note that these mechanisms are quite different from how genetic drift would affect the rate of evolutionary change. Genetic drift reduces the rate of evolutionary change but does so by reducing additive genetic variance, thereby making selection less efficient. For example, in the breeders equation $R=h^{2} S$, where $R$ is the change in a quantitative trait, $h^{2}$ is heritability, and $S$ is the selection differential, genetic drift will reduce $R$ by reducing herita- 
bility. Our results reveal that adding nongenetic variation in the form of individual heterogeneity decreases both heritability and the selection differential, resulting in slowerthan-expected phenotypic change compared with what would be observed if the impact occurred through heritability only, as it would be for genetic drift. More generally, by explicitly accounting for individual heterogeneity in population models, it is straightforward to mechanistically link heritability and selection in models of phenotypic change.

The ecological response to nongenetic variation in our model depended a great deal on the covariance structure of the traits when they were generated from nongenetic sources of variation. Negative correlations among the traits caused a decrease in population growth, whereas uncorrelated or positive covariance caused an increase in population growth. The ecological dynamics responded differently than evolutionary dynamics to nongenetic variation because the processes that reduce relative fitness among genotypes have different impacts on the average fitness of a group of genotypes. The effect of having a concave fitness function reduces the average fitness, resulting in slower population growth and lower density. In contrast, the frailty effect increases the average fitness, resulting in faster population growth and higher density. The net effect found in our simulations (fig. 5) emerges from the fact that the different covariance structures impact the relative strength of these two processes. For example, if there is positive genetic trait covariance, there is a reasonable chance that a genotype will have low reproduction, development, and survivorship - all of which lead to strong frailty effects. If there is negative genetic covariance, then the frailty effect is weaker because it is unlikely that all traits will be simultaneously low.

An advantage of using a model to scale from vital rates to population dynamics is that it allows us to evaluate the predicted biological significance of observed life-history traits. For example, manipulating food quality in our experiments had a substantial impact on the variance from both nongenetic and genetic sources (fig. 2) and on the covariance structure from nongenetic sources (fig. 3). We can use these data to evaluate the potential impact of foodquality changes on biological dynamics by studying how genetic versus nongenetic sources of individual heterogeneity scale up to cause change at the population level. We found that despite large changes in life-history trait covariance across the gradient of food quality, the predicted relative importance of the different sources of individual heterogeneity to biological dynamics changed much less (fig. 6). Nongenetic trait variation is predicted to have a positive impact on ecological dynamics that becomes more important as food quality decreases. Increasing genetic trait variation is also predicted to increase population growth, but to a lesser degree than nongenetic variation (fig. 6B).
Increasing genetic variation increases the rate of evolution (by increasing fitness variance) across all quality levels, whereas increasing nongenetic variation slows the rate of evolution (fig. 6D). While the particular quantitative conclusions of this analysis are dependent on the model being a good description of the biology of Daphnia, the overall message of figures 5 and 6 is that increasing the amount of nongenetic variation in Daphnia is predicted to result in faster population growth while simultaneously reducing the rate of evolution across the full range of food quality.

Parthenogenetic and clonal organisms provide a rare opportunity to evaluate the relative importance of genetic versus nongenetic sources of variation in life-history traits. Our experiments found that there was substantial variation in Daphnia life-history traits, even under carefully controlled food environments, and unexpectedly found that most of this variation is due to nongenetic sources rather than genetic sources or individual stochasticity. Naturally, these results will help guide the development of mathematical models that incorporate demographic heterogeneity in Daphnia, which is a classic model organism for studying the scaling of life-history traits to biological dynamics (e.g., Nelson et al. 2007; McCauley et al. 2008; Ananthasubramaniam et al. 2011). More generally, however, the importance of identifying the sources of trait variation centers around the distinct impact that each has on ecological and evolutionary dynamics - increasing genetic variation causes evolution to speed up, whereas increasing nongenetic variation causes evolution to slow down. Since the life-history traits and covariance structures investigated in our model are common to many taxa, these results are likely relevant for a wide range of organisms.

\section{Acknowledgments}

We thank S. French for assistance with conducting the experiments and O. Bjørnstad for his deep archival services. This research was funded by a Natural Sciences and Engineering Research Council grant to W.A.N.

\section{Literature Cited}

Acker, P., A. Robert, R. Bourget, and B. Colas. 2014. Heterogeneity of reproductive age increases the viability of semelparous populations. Functional Ecology 28:458-468.

Ananthasubramaniam, B., R. M. Nisbet, W. A. Nelson, E. McCauley, and W. S. C. Gurney. 2011. Stochastic growth reduces population fluctuations in Daphnia-algal systems. Ecology 92:362-372.

Baird, D. J., I. Barber, and P. Calow. 1990. Clonal variation in general responses of Daphnia magna Straus to toxic stress. I. Chronic lifehistory effects. Functional Ecology 4:399-407.

Barata, C., and D. J. Baird. 1998. Phenotypic plasticity and constancy of life-history traits in laboratory clones of Daphnia magna Straus: effects of neonatal length. Functional Ecology 12:442-452. 
Beckerman, A., T. G. Benton, E. Ranta, V. Kaitalac, and P. Lundberg. 2002. Population dynamic consequences of delayed life-history effects. Trends in Ecology and Evolution 17:263-269.

Benton, T. G., S. J. Plaistow, and T. N. Coulson. 2006. Complex population dynamics and complex causation: devils, details and demography. Proceedings of the Roval Society B 273:1173-1181.

Bolnick, D. I., P. Amarasekare, M. S. Araújo, R. Bürger, J. M. Levine, M. Novak, V. H. W. Rudolf, S. J. Schreiber, M. C. Urban, and D. A. Vasseur. 2011. Why intraspecific trait variation matters in community ecology. Trends in Ecology and Evolution 26:183192.

Boyce, M. S. 1977. Population growth with stochastic fluctuations in the life table. Theoretical Population Biology 12:366-373.

Briggs, C. J., S. M. Sait, M. Begon, D. J. Thompson, and H. C. J. Godfray. 2000. What causes generation cycles in populations of stored-product moths? Lournal of Animal Ecology 69:352366.

Browne, R. A., V. Moller, V. E. Forbes, and M. H. Depledge. 2002. Estimating genetic and environmental components of variance using sexual and clonal Artemia. Journal of Experimental Marine Biology and Ecology 267:107-119.

Cam, E., W. A. Link, E. G. Cooch, J.-Y. Monnat, and E. Danchin. 2002. Individual covariation in life-history traits: seeing the trees despite the forest. American Naturalist 159:96-105.

Caswell, H. 2009. Stage, age and individual stochasticity in demography. Oikos 118:1763-1782.

Claessen, D., A. M. de Roos, and L. Persson. 2000. Dwarfs and giants: cannibalism and competition in size-structured populations. American Naturalist 155:219-237.

Conner, M. M., and G. C. White. 1999. Effects of individual heterogeneity in estimating the persistence of small populations. Natural Resource Modeling 12:109-127.

Coulson, T. G., E. A. Catchpole, S. D. Albon, B. J. T. Morgan, J. M. Pemberton, T. H. Clutton-Brock, M. J. Crawley, and B. T. Grenfell. 2001. Age, sex, density, winter weather, and population crashes in Soay sheep. Science 292:1528-1531.

De Meester, L. 1995. Life history characteristics of Daphnia magna clones differing in phototactic behaviour. Hydrobiologia 307:167175.

de Roos, A. M., and L. Persson. 2013. Population and community ecology of ontogenetic development. Princeton University Press, Princeton, NJ.

Ebert, D. 1991. The effect of size at birth, maturation threshold and genetic differences on the life-history of Daphnia magna. Oecologia 86:243-250.

-1994. A maturation size threshold and phenotypic plasticity of age and size at maturity in Daphnia magna. Oikos 69:309317.

Elser, J. J., T. Andersen, J. S. Baron, A. K. Bergström, M. Jansson, M. Kyle, K. R. Nydick, L. Steger, and D. O. Hessen. 2009. Shifts in lake $\mathrm{N}: \mathrm{P}$ stoichiometry and nutrient limitation driven by atmospheric nitrogen deposition. Science 326:835-837.

Epp, G. T. 1996. Clonal variation in the survival and reproduction of Daphnia pulicaria under low-food stress. Freshwater Biology 35:1-10.

Fisher, R. A. 1930. The genetical theory of natural selection. Oxford University Press, Oxford.

Forbes, V. E., V. Moller, and M. H. Depledge. 1995. Intrapopulation variability in sublethal response to heavy metal stress in sexual and asexual gastropod populations. Functional Ecology 9:477484.

Fox, G. A., B. E. Kendall, J. W. Fitzpatrick, and G. E. Woolfenden. 2006. Consequences of heterogeneity in survival probability in a population of Florida scrub-jays. Iournal of Animal Ecology 75:921-927.

Gilbert, J. J., and T. Schroder. 2007. Intraclonal variation in propensity for mixis in several rotifers: variation among females and with maternal age. Hydrobiologia 593:121-128.

González-Suárez, M., J.-F. Le Gaillard, and D. Claessen. 2011. Population and life-history consequences of within-cohort individual variation. American Naturalist 178:525-537.

Grimm, V., and J. Uchmański. 2002. Individual variability and population regulation: a model of the significance of within-generation density dependence. Oecologia 131:196-202.

Guildford, S. J., and R. E. Hecky. 2000. Total nitrogen, total phosphorus, and nutrient limitation in lakes and oceans: is there a common relationship? Limnology and Oceanography 45:1213-1223.

Hadfield, J. D. 2010. MCMC methods for multi-response generalised linear mixed models: the MCMCglmm R package. Iournal of Statistical Software 33:1-22.

Hansen, T. F., C. Pélabon, and D. Houle. 2011. Heritability is not evolvability. Evolutionary Ecology 38:258-277.

Hebert, P. D. N., and M. J. Beaton. 1993. Methodologies for allozyme analysis using cellulose acetate electrophoresis. Helena Laboratories, Beaumont, TX.

Kendall, B. E., and G. A. Fox. 2002. Variation among individuals and reduced demographic stochasticity. Conservation Biology 16:109116.

. 2003. Unstructured individual variation and demographic stochasticity. Conservation Biology 17:1170-1172.

Kendall, B. E., G. A. Fox, M. Fujiwara, and T. M. Nogeire. 2011. Demographic heterogeneity, cohort selection, and population growth. Ecology 92:1985-1993.

Kilham, S. S., D. A. Kreeger, S. G. Lynn, C. E. Goulden, and L. Herrera. 1998. COMBO: a defined freshwater culture medium for algae and zooplankton. Hydrobiologia 377:147-159.

Latta, L. C., IV, M. Peacock, D. J. Civitello, J. L. Dudycha, J. M. Meik, and S. Schaack. 2015. The phenotypic effects of spontaneous mutations in different environments. American Naturalist 185:243252.

Lehman, J. T., and C. D. Sandgren. 1982. Phosphorus dynamics of the procaryotic nannoplankton in a Michigan lake. Limnology and Oceanography 27:828-838.

Lindström, J., and H. Kokko. 2002. Cohort effects and population dynamics. Ecology Letters 5:338-344.

Lynch, M. 1988. The rate of polygenic mutation in Daphnia. Evolution 38:465-482.

McCauley, E., W. A. Nelson, and R. M. Nisbet. 2008. Smallamplitude cycles emerge from stage-structured interactions in Daphnia-algal systems. Nature 455:1240-1243.

Melbourne, B. A., and A. Hastings. 2008. Extinction risk depends strongly on factors contributing to stochasticity. Nature 454:100103.

Metcalf, C. J. E., and S. Pavard. 2007. Why evolutionary biologists should be demographers. Trends in Ecology and Evolution 22: 205-212.

Mousseau, T. A., and D. A. Roff. 1987. Natural selection and the heritability of fitness components. Heredity 59:181-197. 
Nelson, W. A., O. N. Bjørnstad, and T. Yamanaka. 2013. Recurrent insect outbreaks caused by temperature-driven changes in system stability. Science 341:796-799.

Nelson, W. A., E. McCauley, and R. M. Nisbet. 2007. Stagestructured cycles generate strong fitness-equalizing mechanisms. Evolutionary Ecology 21:499-515.

Neubert, M. G., and H. Caswell. 2000. Density-dependent vital rates and their population dynamic consequences. Journal of Mathematical Biology 41:103-121.

Olijnyk, A. O., and W. A. Nelson. 2013. Positive phenotypic correlations among life-history traits remain in the absence of differential resource ingestion. Functional Ecology 27:165-172.

Paloheimo, J. E., S. J. Crabtree, and W. D. Taylor. 1982. Growth model of Daphnia. Canadian Journal of Fisheries and Aquatic Sciences 39:598-606.

Pelletier, F., T. Clutton-Brock, J. Pemberton, S. Tuljapurkar, and T. Coulson. 2007. The evolutionary demography of ecological change: linking trait variation and population growth. Science 315:15711574.

Pelletier, F., K. Moyes, T. H. Clutton-Brock, and T. Coulson. 2012. Decomposing variation in population growth into contributions from environment and phenotypes in an age-structured population. Proceedings of the Roval Society B 279:394-401.

Persson, L., P.-A. Amundsen, A. M. de Roos, A. Klemetsen, R. Knudsen, and R. Primicerio. 2007. Culling prey promotes predator recovery: alternative states in a whole-lake experiment. Science 316:1743-1746.

Persson, L., P. Byström, and E. Wahlström. 2000. Cannibalism and competition in Eurasian perch: population dynamics of an ontogenetic omnivore. Ecology 81:1058-1071.

Pietrzak, B. 2011. Interclonal differences in age-specific performance in Daphnia magna. Journal of Limnology 70:345-352.

Plath, K., and M. Boersma. 2001. Mineral constraints of zooplankton: stoichiometric constraints and optimal foraging. Ecology 82:1260-1269.

Price, T., and D. Schluter. 1991. On the low heritability of life-history traits. Evolution 45:853-861.

Pya, N., and S. N. Wood. 2015. Shape constrained additive models. Statistics and Computing 25:543-559.

R Core Team. 2014. R: a language and environment for statistical computing. R Foundation for Statistical Computing, Vienna. http://www.R-project.org.

Reznick, D., L. Nunney, and A. Tessier. 2000. Big houses, big cars, superfleas and the costs of reproduction. Trends in Ecology and Evolution 15:421-425.

Sgrò, C. M., and A. A. Hoffmann. 2004. Genetic correlations, tradeoffs and environmental variation. Heredity 93:241-248.
Singh, B. K., J. Subba Rao, R. Ramaswamy, and S. Sinha. 2004. The role of heterogeneity on the spatiotemporal dynamics of hostparasite metapopulation. Ecological Modelling 180:435-443.

Spitze, K., J. Burnson, and M. Lynch. 1991. The covariance structure of life-history characters in Daphnia pulex. Evolution 45:1081-1090.

Sterner, R. W., and D. O. Hessen. 1994. Algal nutrient limitation and the nutrition of aquatic herbivores. Annual Review of Ecology and Systematics 25:1-29.

Stover, J. P., B. E. Kendall, and G. A. Fox. 2012. Demographic heterogeneity impacts density-dependent population dynamics. Theoretical Ecology 5:297-309.

Tessier, A., and N. L. Consolatti. 1989. Variation in offspring size in Daphnia and consequences for individual fitness. Oikos 56:269276.

Urabe, J., and Y. Watanabe. 1992. Possibility of $\mathrm{N}$ or P limitation for planktonic cladocerans: an experimental test. Limnology and Oceanography 37:244-251.

van Daalen, S., and H. Caswell. 2015. Lifetime reproduction and the second demographic transition: stochasticity and individual variation. Demographic Research 33:561-588.

van Donk, E., M. Lürling, D. O. Hessen, and G. M. Lockhorst. 1997. Altered cell wall morphology in nutrient-deficient phytoplankton and its impact on grazers. Limnology and Oceanography 42:357364.

Vanni, M. J. 1987. Colonization dynamics and life history traits of seven Daphnia pulex genotypes. Oecologia 72:263-271.

van Noordwijk, A. J., and G. de Jong. 1986. Acquisition and allocation of resources: their influence on variation in life-history tactics. American Naturalist 128:137-142.

Vindenes, Y., S. Engen, and B. E. Sæther. 2008. Individual heterogeneity in vital parameters and demographic stochasticity. American Naturalist 171:455-467.

Vindenes, Y., and Ø. Langangen. 2015. Individual heterogeneity in life histories and eco-evolutionary dynamics. Ecology Letters 18:417-432.

Yampolsky, L. Y., and S. M. Scheiner. 1994. Developmental noise, phenotypic plasticity, and allozyme heterozygosity in Daphnia. Evolution 48:1715-1722.

\section{References Cited Only in the Online Appendixes}

Guisande, C., and Z. M. Głiwicz. 1992. Egg size and clutch size in two Daphnia species grown at different food levels. Lournal of Plankton Research 14:997-1007.

Associate Editor: Daniel I. McBolnick Editor: Yannis Michalakis 